\title{
Maximization of Spatial Charge Density: An Approach to Ultrahigh Energy Density of Capacitive Charge Storage
}

\author{
Hongyun Ma, Hongwu Chen, Mingmao Wu, Fengyao Chi, Feng Liu, * Jiaxin Bai, Huhu Cheng, \\ Chun Li, and Liangti $Q u^{*}$
}

\begin{abstract}
Capacitive energy storage has advantages of high power density, long lifespan, and good safety, but is restricted by low energy density. Inspired by the charge storage mechanism of batteries, a spatial charge density (SCD) maximization strategy is developed to compensate this shortage by densely and neatly packing ionic charges in capacitive materials. A record high SCD (ca. $\left.550 \mathrm{Ccm}^{-3}\right)$ was achieved by balancing the valance and size of charge-carrier ions and matching the ion sizes with the pore structure of electrode materials, nearly five times higher than those of conventional ones $\left(\mathrm{ca} .120 \mathrm{C} \mathrm{cm}^{-3}\right)$. The maximization of SCD was confirmed by Monte Carlo calculations, molecular dynamics simulations, and in situ electrochemical Raman spectroscopy. A full-cell supercapacitor was further constructed; it delivers an ultrahigh energy density of $165 \mathrm{Wh} \mathrm{L}^{-1}$ at a power density of $150 \mathrm{WL}^{-1}$ and retains $120 \mathrm{Wh} \mathrm{L}^{-1}$ even at $36 \mathrm{~kW} \mathrm{~L}^{-1}$, opening a pathway towards high-energy-density capacitive energy storage.
\end{abstract}

\section{Introduction}

Electrochemical energy storage (EES) devices with excellent integrated performances, such as high energy/power density, long lifespan, good security, and low cost, are urgently desired to adapt to the fast development of portable electronics and electric vehicles. ${ }^{[1-3]}$ According to the different charge storage mechanisms, EES devices can be basically divided into batteries and supercapacitors. ${ }^{[4]}$ Batteries store charge via reversible bulk phase redox reaction where bare ions are employed as the charge carriers, ${ }^{[5,6]}$ whereas the charge storage of supercapacitors relies on the fast surface

[*] H. Ma, H. Chen, M. Wu, F. Chi, Prof. C. Li, Prof. L. Qu

Department of Chemistry, Tsinghua University

Beijing 100084 (P. R. China)

E-mail: lqu@mail.tsinghua.edu.cn

Prof. F. Liu

State Key Laboratory of Nonlinear Mechanics

Institute of Mechanics, Chinese Academy of Sciences

Beijing 100190 (P. R. China)

E-mail: liufeng@imech.ac.cn

J. Bai, H. Cheng, Prof. L. Qu

Key Laboratory for Advanced Materials Processing Technology, Ministry of Education of China; State Key Laboratory of Tribology, Department of Mechanical Engineering, Tsinghua University Beijing 100084 (P. R. China)

(2) Supporting information and the ORCID identification number(s) for (D) the author(s) of this article can be found under: https://doi.org/10.1002/anie.202005270. adsorption/desorption of solvated ions. ${ }^{[7,8]}$ By contrast, the surface charge storage mechanism provides supercapacitors with high power density and long lifespan, but simultaneously restricts their energy density. ${ }^{[9]}$ Therefore, plenty of efforts have recently been made to increase the effective surface area of the capacitive materials, and/or introduce pseudo-capacitive reactions to improve the charge density stored at unit surface area ${ }^{[10-12]}$ Though these approaches have made great progress, the energy densities of supercapacitors (ca. $20 \mathrm{Wh} \mathrm{kg}^{-1}$ ) are still much lower than those of batteries (ca. $200 \mathrm{Wh} \mathrm{kg}^{-1}$ ). Originally, the main shortcoming of the capacitive energy storage mechanism is its low spatial charge density (SCD), which unfortunately has rarely received proper attention.

For battery-type materials, bare ions are densely and neatly packed within them (Figure $1 \mathrm{a}$ ), thus possessing high SCDs and corresponding high energy densities. But the sluggish solid-phase diffusion of bare ions and concomitant ion solvation/desolvation processes greatly restrict their power densities. ${ }^{[5,6,13]}$ By comparison, capacitive materials deliver ultrahigh power densities owing to the fast liquidphase ion transport within them and the elimination of sluggish solvation/desolvation processes. ${ }^{[14]}$ However, in this case, huge solvated ions are loosely and randomly distributed within the active materials (Figure 1b), which severely limits their SCDs and corresponding energy densities. ${ }^{[15,16]}$ In light of this, densely and neatly packing solvated ions in one capacitive material will be an effective approach to maximizing the SCD of capacitive energy storages, and thus achieving both high energy and high power performances simultaneously (Figure $1 \mathrm{c}$ ).

Herein, we develop a SCD maximization strategy to improve the energy density of capacitive charge storage, where ionic charges are densely and neatly packed in electrode materials. By balancing the valance and size of charge-carrier ions and matching the ion sizes with the pore structure of electrode materials, a record high SCD of about $550 \mathrm{Ccm}^{-3}$ was achieved, which is nearly five times higher than those of conventional ones (ca. $120 \mathrm{Ccm}^{-3}$ ). Monte Carlo calculation, molecular dynamics simulation, and in situ electrochemical Raman spectroscopy were employed to track the electrochemical behaviors of various multivalent metal ions in capacitive materials, and their partial dehydration processes were found to be an essential procedure to match and enter sub-nano pores. On these bases, a zinc-ion capacitor was constructed and it delivers an ultrahigh energy density of $165 \mathrm{WhL}^{-1}$ at a power density of $150 \mathrm{WL}^{-1}$, and retains a high energy density of $120 \mathrm{WhL}^{-1}$ even at an ultrahigh power 
a

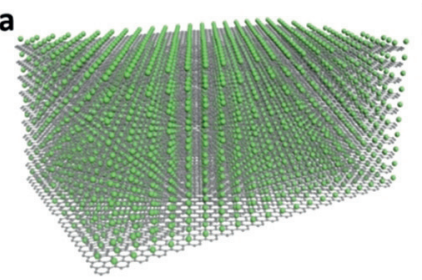

b

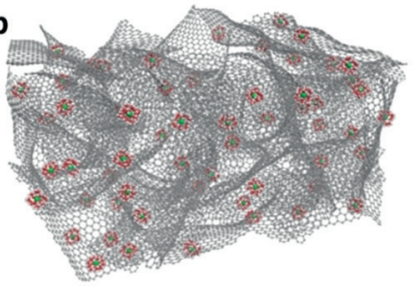

C

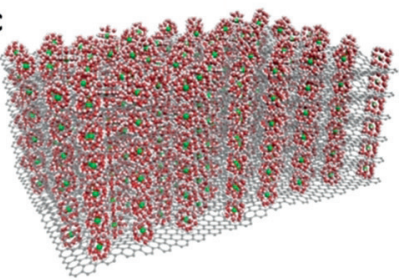

d

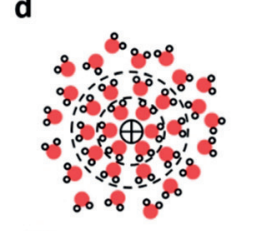

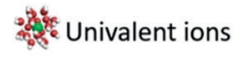

ate

और्ष
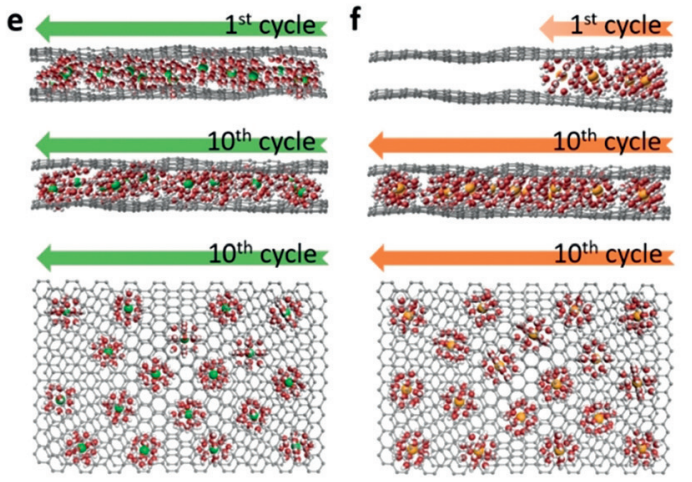

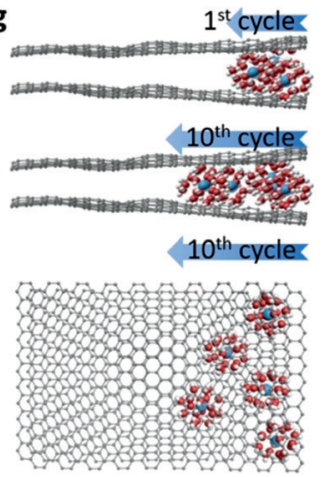

Figure 1. Illustration of the SCD maximization strategy. a) Typical configurations of bare ions in battery-type material and b) solvated ions in capacitive material. c) Densely and neatly packing solvated ions within HOPC to achieve ultrahigh SCD. d) Typical configuration of hydrated cation, with circles representing hydration shells (top); configurations of various ions with different radii (bottom). e)-g) Distributions of different ions in compact HOPC before (1st cycle) and after (10th cycle) electrochemical activations: e) univalent, f) divalent, and g) trivalent ions.

density of $36 \mathrm{kWL}^{-1}$. These excellent performances strongly demonstrate the validity and rationality of the proposed SCD maximization strategy to enhance the energy density of capacitive charge storage.

\section{Results and Discussion}

As mentioned above, the charge storage of supercapacitor is closely relied on electrode/electrolyte interface, namely the formation of electrochemical double layers. ${ }^{[17,18]}$ Considering that the charge-carrier ions are adsorbed on the active surface within the electrode material, the microstructure of the electrode material will directly dominate the distribution of charge-carrier ions in one electrode. ${ }^{[19]}$ Therefore, an ordered and compact carbon scaffold is indispensable and of great importance to densely and neatly pack the solvated chargecarrier ions. Accordingly, a highly ordered and compact porous carbon (HOPC) was prepared (Supporting Information, Figures S1-S5), as reported in our previous works. ${ }^{[2,21]}$ The highly ordered and compact microstructure provides the HOPC with an ultrahigh volumetric specific surface area (SSA) of $1959 \mathrm{~m}^{2} \mathrm{~cm}^{-3}$ (Supporting Information, Figure S6), which makes HOPC fully capable of storing large amounts of charges in a given space.

Having constructed the HOPC, the next key point is to fill it with as many ionic charges as possible. Compared with commonly used univalent metal ions (for example, $\mathrm{Li}^{+}, \mathrm{Na}^{+}$, and $\mathrm{K}^{+}$ions), multivalent metal ions (for example, $\mathrm{Zn}^{2+}$, $\mathrm{Mg}^{2+}, \mathrm{Ca}^{2+}$, and $\mathrm{Al}^{3+}$ ions) are more competent charge carriers because of their multi-electron electrochemistry. ${ }^{[22,23]}$ However, owing to the larger hydrated radii of higher valence ions (Figure 1d; Supporting Information, Table S1), there will be a trade-off between the valence and hydrated radius of the charge-carrier ions for compact HOPC electrode. In fact, we found that univalent ions $\left(\mathrm{Li}^{+}, \mathrm{Na}^{+}\right.$, and $\mathrm{K}^{+}$ions) can get access to the whole active surface area within HOPC even at the first charging/discharging cycle (Figure 1e). Divalent ions $\left(\mathrm{Mg}^{2+}\right.$, and $\mathrm{Zn}^{2+}$ ions) can also reach all the active surface area within HOPC upon electrochemical activations (Figure $1 \mathrm{f})$. However, for the trivalent metal ion $\left(\mathrm{Al}^{3+}\right.$ ion), owing to its large hydrated radius and high hydration free energy, it cannot effectively infiltrate the HOPC even upon repeated activations (Figure 1g). As a result, an ultrahigh SCD of about $550 \mathrm{Ccm}^{-3}$ was achieved on the basis of divalent ions (Supporting Information, Table S2). Remarkably, this value is nearly five times higher than those of activated carbon based conventional systems (ca. $120 \mathrm{Ccm}^{-3}$ ). Also, this ion sieving effect is virtually an effective criterion for maximizing the SCD of one electrode material, which will be discussed in detail in the following section.

The electrochemical behaviors of different charge-carrier metal ions in HOPC were systematically study by cyclic voltammetry (CV) and electrochemical impedance spectroscopy (EIS). For comparison, activated carbon (AC, YP-50F herein) based systems were also studied given that $\mathrm{AC}$ is the most commonly used capacitive electrode material with the merits of high SSA, hierarchical porous structure, and good conductivity (Supporting Information, Figure S7). As shown in Figure $2 \mathrm{a}$ and the Supporting Information, Figure S8, the specific capacitances of AC based electrodes increase linearly with the valences of employed metal ions. This result suggests that AC can effectively accommodate all kinds of multivalent ions, and the multivalent ions with higher valences contribute higher specific capacitances. But for the highly ordered and compact HOPC, a maximum specific capacitance is achieved 

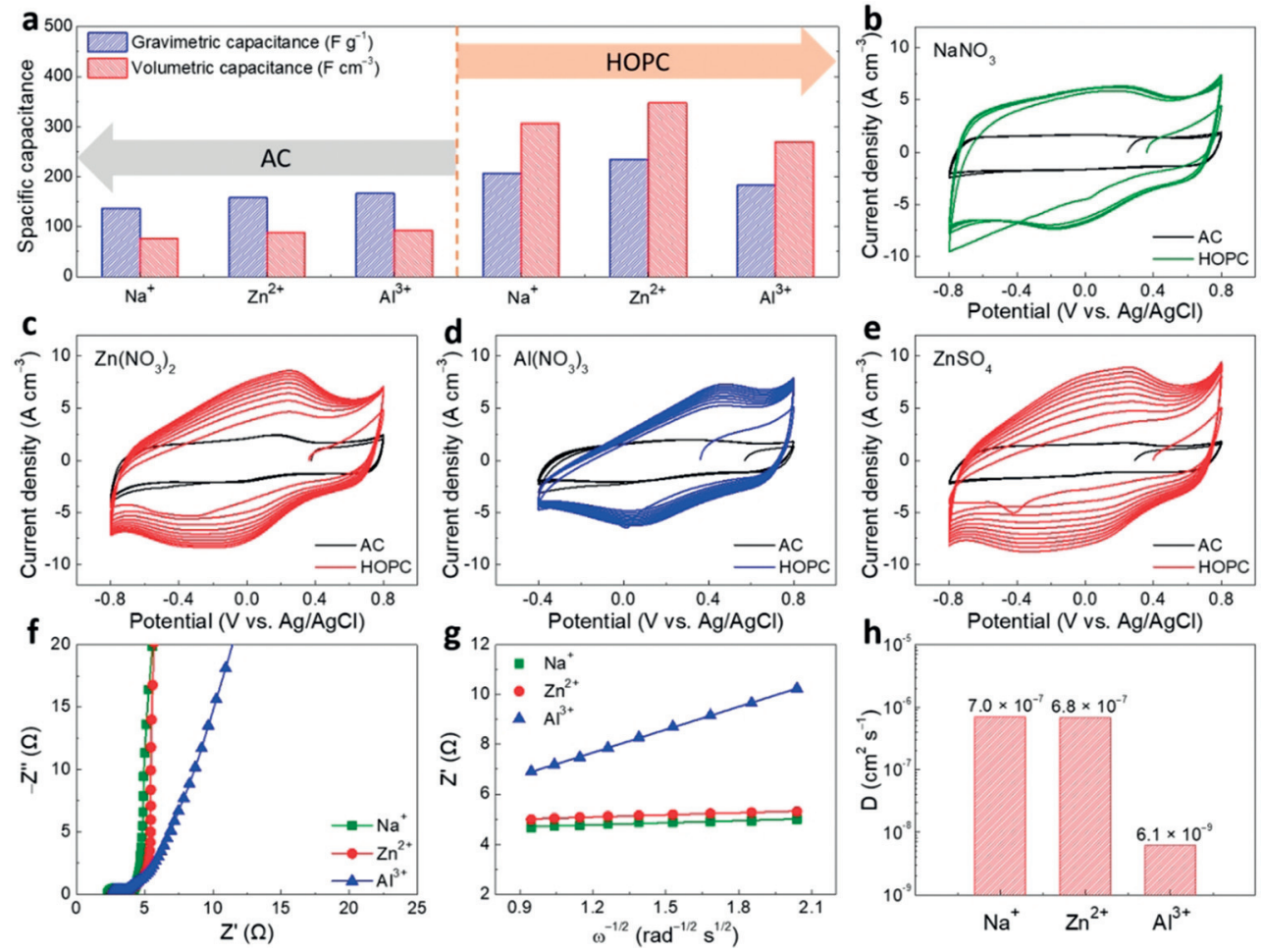

Figure 2. Electrochemical behaviors of different ions in $A C$ and HOPC. a) Gravimetric and volumetric capacitances of $A C$ and $H O P C$ at $1 \mathrm{Ag}^{-1}$. b)-e) The first ten $\mathrm{CV}$ curves of $\mathrm{AC}$ and HOPC in different electrolytes: b) $\left.\mathrm{NaNO}_{3}, \mathrm{c}\right) \mathrm{Zn}\left(\mathrm{NO}_{3}\right)_{2}$, d) $\mathrm{Al}\left(\mathrm{NO}_{3}\right)_{3}$, e) $\mathrm{ZnSO}$. f) Nyquist plots of HOPC in different electrolytes, g) corresponding linear plots of Real resistances $\left(Z^{\prime}\right)$ against angular frequencies $\left(\omega^{-1 / 2}\right)$ in the low frequency region, and h) derived ion diffusion coefficients.

when taking divalent ions as the charge carriers. Notably, owing to its high packing density of $1.48 \mathrm{~g} \mathrm{~cm}^{-3}, \mathrm{HOPC}$ possesses an ultrahigh volumetric surface area of $1959 \mathrm{~m}^{2} \mathrm{~cm}^{-3}$, which is over two times higher than that of AC $\left(938 \mathrm{~m}^{2} \mathrm{~cm}^{-3}\right.$, Supporting Information, Figure S6). The high volumetric surface area provides HOPC with much more available space or active surface area to store ionic charges, rather than to accommodate inactive free electrolytes.

The CV technique was subsequently carried out to investigate the ion sieving effect of HOPC and, meanwhile, to fully match the pores of HOPC with the charge-carrier ions. As shown in Figure $2 \mathrm{~b}$ and the Supporting Information, Figures S9, S10, the first ten CV curves of both AC and $\mathrm{HOPC}$ are well overlapped, indicating that the pores of both $\mathrm{AC}$ and $\mathrm{HOPC}$ are large enough to accommodate univalent ions (here, $\mathrm{Na}^{+}$ion was taken as an example). Notably, the much larger response current $\left(\mathrm{Acm}^{-3}\right)$ of HOPC electrode reflects a higher SCD within HOPC. When changing the charge carriers from univalent ion $\left(\mathrm{Na}^{+}\right.$ion) to divalent ion $\left(\mathrm{Zn}^{2+}\right.$ ion), AC shows almost the same electrochemical behavior (Figure 2c). However, an obvious electrochemical activation phenomenon appears for HOPC, indicating that divalent ions cannot spontaneously infiltrate fresh HOPC electrode but can reach all the active surface area inside HOPC upon electrochemical activations. For the trivalent $\mathrm{Al}^{3+}$ ion, the electrochemical activation process still exists for HOPC but is substantially restricted, suggesting that trivalent $\mathrm{Al}^{3+}$ ion cannot effectively infiltrate HOPC even upon repeated activations (Figure $2 \mathrm{~d}$; Supporting Information, Figure S11).

Above $\mathrm{CV}$ studies show that compact HOPC exhibits an obvious sieving effect on charge-carrier metal ions, and divalent ions can well match the pore structure of HOPC and thus deliver the highest SCDs among other metal ions. This conclusion was further confirmed by substitute divalent $\mathrm{Zn}^{2+}$ ion with divalent $\mathrm{Mg}^{2+}$ ion. As shown in the Supporting Information, Figure $\mathrm{S} 12$, the $\mathrm{Mg}^{2+}$ ion based system shows a similar electrochemical behavior with $\mathrm{Zn}^{2+}$ ion based system, which results from the identical valences and almost equivalent radii of $\mathrm{Mg}^{2+}$ and $\mathrm{Zn}^{2+}$ ions. Additionally, the influence of counterions was also checked (Figure 2e; Supporting Information, Figure S13). Both HOPC and AC show almost unchanged electrochemical behaviors after replacing the $\mathrm{NO}_{3}{ }^{-}$ion with the $\mathrm{SO}_{4}{ }^{2-}$ ion, indicating the negligible effects of anions in this case. This result mainly originates from the cation-dominated charge storage mechanisms of HOPC and AC on the one hand (Supporting Information, Figure S14), and the relatively small hydrated radii of anions on the other hand $\left(0.340\right.$ and $0.379 \mathrm{~nm}$ for $\mathrm{NO}_{3}{ }^{-}$and $\mathrm{SO}_{4}{ }^{2-}$ ions, respectively).

The above-mentioned ion sieving effect and pore and ion matching principle were further supported by the EIS studies. After enduring electrochemical activation in $\mathrm{Zn}^{2+}$ electrolyte, HOPC shows an ideal capacitive behavior with nearly vertical Nyquist plots in the low frequency (Figure 2f). This behavior is similar to that of $\mathrm{HOPC}$ in $\mathrm{Na}^{+}$electrolyte, and those of $\mathrm{AC}$ in $\mathrm{Na}^{+}, \mathrm{Zn}^{2+}$, and $\mathrm{Al}^{3+}$ electrolytes (Supporting Information, 

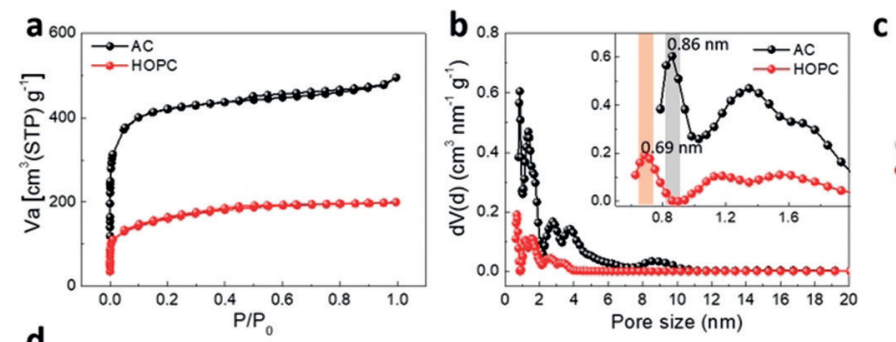

C
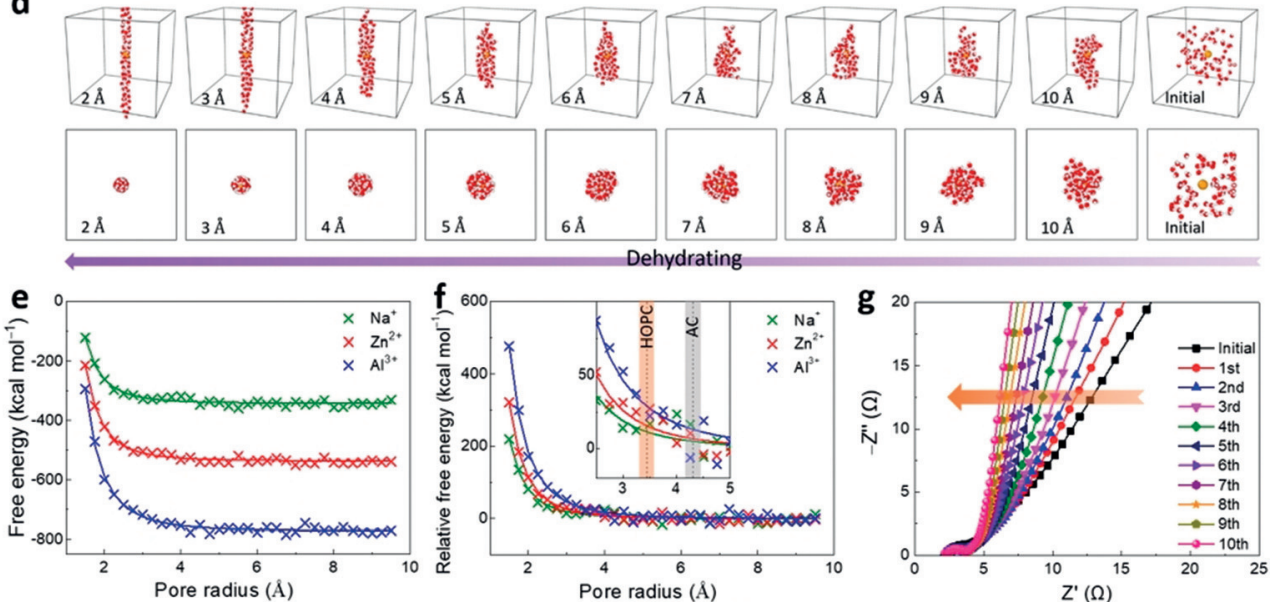

h
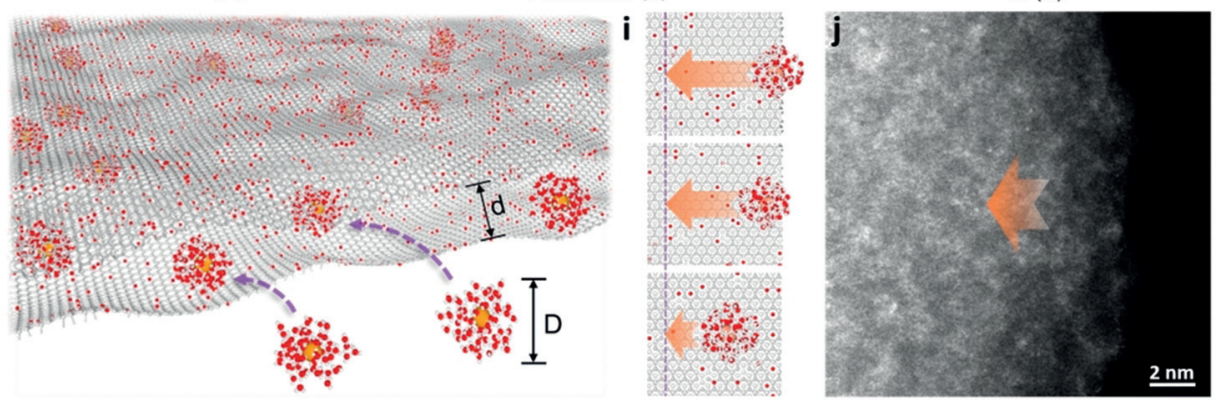

Figure 3. Investigations of the ion sieving effect of HOPC and corresponding pore and ion matching principle. a) $\mathrm{N}_{2}$ adsorption/desorption isotherms of AC and HOPC and b) corresponding PSD curves. c) Illustration of the partial dehydration of charge-carrier ions. d) Calculated configurations of $\mathrm{Zn}^{2+}$ ion within gradually decreased pores. e) Free energy evolution with respect to pore radius. f) Relative free energy evolution with respect to pore radius. g) Real-time EIS Nyquist plots of HOPC upon electrochemical activation. h) Comprehensive picture of the electrochemical behavior of $\mathrm{Zn}^{2+}$ ion in compact HOPC. i) Entering pore process upon partial dehydration. j) HAADF-STEM image of HOPC at fully discharged state, with dense bright spots showing the $\mathrm{Zn}^{2+}$ ions stored in HOPC.

Figure S15). However, the Nyquist plots of HOPC in $\mathrm{Al}^{3+}$ electrolyte show an obvious Warburg region, reflecting the sluggish ion transport in this case. These results further demonstrate that divalent metal ions are the most ideal charge carriers for the compact HOPC, where high SCD and fast charge transport can be achieved simultaneously. Furthermore, the ion diffusion coefficients in HOPC were measured via the EIS technique (Figure $2 \mathrm{~g}$,h; Supporting Information, Figure S16). ${ }^{[21]}$ The divalent $\mathrm{Zn}^{2+}$ ion exhibits a high ion diffusion coefficient of $6.8 \times 10^{-7} \mathrm{~cm}^{2} \mathrm{~s}^{-1}$, which is close to that of univalent $\mathrm{Na}^{+}$ion $\left(7.0 \times 10^{-7} \mathrm{~cm}^{2} \mathrm{~s}^{-1}\right)$ and two orders of magnitude higher than that of trivalent $\mathrm{Al}^{3+}$ ion $\left(6.1 \times 10^{-9} \mathrm{~cm}^{2} \mathrm{~s}^{-1}\right)$, further reflecting that divalent metal ions can well match the pores of HOPC.

The above results have shown that divalent ions can well match the pore structure of HOPC and hence achieve ultrahigh SCDs. Theoretical studies were carried out to get a deep insight into this point. As shown in Figure $3 \mathrm{a}$, the nitrogen adsorption/desorption curves of both HOPC and AC exhibit a characteristic of type I isotherms with type H4 hysteresis loops, indicating the coexistence of micropores $(<2 \mathrm{~nm})$ and mesopores $(2-50 \mathrm{~nm})$ in both HOPC and AC. ${ }^{[24-26]}$ To get more details, the pore size distribution (PSD) of both HOPC and AC were calculated via the stateof-the-art density functional theory (DFT). As shown in Figure $3 \mathrm{~b}$ and the inset, the PSD peaks of the smallest pores for $\mathrm{HOPC}$ and $\mathrm{AC}$ are centered at $0.69 \mathrm{~nm}$ and $0.86 \mathrm{~nm}$ respectively in diameter, namely at $0.345 \mathrm{~nm}$ and $0.430 \mathrm{~nm}$ respectively in radius. Obviously, the smallest pores of HOPC are smaller than almost all the charge-carrier ions involved in this work (Supporting Information, Table S1), though they contribute a substantial part of active SSA to HOPC. Therefore, it is reasonable to infer that large charge-carrier ions shall undergo a partial dehydration procedure when they are entering the smallest pores of HOPC (Figure $3 \mathrm{c}) .^{[8,27-31]}$

To track this process, Monte Carlo simulations were carried out and the calculation models are shown in the Supporting Information, Figures S17, S18, where a bare ion is 
fixed in the center of a cylinder pore and surrounded by a hydration shell containing 72 water molecules. ${ }^{[32,33]}$ Herein, the dehydration of target ions was abstracted and described as the pore size effect on the deformation of hydration shell, which results from the constrain of water molecules by gradually decreased cylinder wall (Figure 3d; Supporting Information, Figures S19-S24). In this way, the system free energy with respect to the pore radius was calculated, as depicted in Figure 3 e. With decreasing pore radius, the pore size effect gradually appears and system free energy gradually increases for all three ions. Although the general trend is similar, the increasing rate is quite different, which shall account for the ion sieving effect of HOPC and corresponding pore and ion matching principle. For a more intuitive comparison, Function $F(r)=\mathrm{a} r^{\mathrm{b}}+\mathrm{c}$ was further used to fit the free energy evolution during the pore radius variation, where $r(\AA)$ is the pore radius and a, b, or c is constant (Supporting Information, Table S3). Subsequently, a relative free energy can be defined as $F_{\mathrm{Rel}}=F(r)-F_{\mathrm{Ref}}$, where $F_{\mathrm{Ref}}$ represents the initial free energy $F(r=10 \AA)$. As shown in Figure $3 \mathrm{f}$, the relative free energy for each ion begins to increase as the pore radius is smaller than $5 \AA$, and the increasing rate for $\mathrm{Al}^{3+}$ ion is much faster than those for $\mathrm{Na}^{+}$ and $\mathrm{Zn}^{2+}$ ions. This phenomenon indicates that it is much more difficult for $\mathrm{Al}^{3+}$ ion to dehydrate to a certain size and then get into a relatively small pore, which is mainly attributed to the much stronger electric field around bare $\mathrm{Al}^{3+}$ ion and thus larger dehydration energy.

The inset in Figure $3 \mathrm{f}$ shows an enlarged vision of the $2.5 \AA<r<5 \AA$ region, which covers the characteristic pore sizes of HOPC (marked as the orange region) and $\mathrm{AC}$ (marked as the gray region). Clearly, there are almost no free energy variations for $\mathrm{Na}^{+}$and $\mathrm{Zn}^{2+}$ ions to get into the pores of $\mathrm{AC}$, and a small free energy increase for $\mathrm{Al}^{3+}$ ion, indicating the ions getting into $\mathrm{AC}$ are $\mathrm{Na}^{+}$ion, $\mathrm{Zn}^{2+}$ ion, and partially dehydrated $\mathrm{Al}^{3+}$ ion, consistent with the radius relationship of $\mathrm{Na}^{+}$ion $(0.358 \mathrm{~nm})<\mathrm{Zn}^{2+}$ ion $(0.430 \mathrm{~nm}) \leq$ $\mathrm{AC}(0.430 \mathrm{~nm})<\mathrm{Al}^{3+}$ ion $(0.475 \mathrm{~nm})$. However, for HOPC, its characteristic pore size $(0.345 \mathrm{~nm})$ is smaller than those of all three ions; therefore, partial dehydration shall be an indispensable procedure for those ions when they are entering HOPC. Unfortunately, unlike $\mathrm{Na}^{+}$and $\mathrm{Zn}^{2+}$ ions, $\mathrm{Al}^{3+}$ ion suffers from a large free energy increase when it is dehydrated to the characteristic pore size of HOPC. This result thus can comprehensively account for the ion sieving effect of HOPC and corresponding pore and ion matching principle.

The electrochemical activation process of HOPC electrode in $\mathrm{Zn}^{2+}$ electrolyte was further comprehended by molecular dynamics simulations. As shown in the Supporting Information, Figure S25, the pillar effect of embedded ions plays a crucial role. Specifically, the embedded ions produce a mechanical support on the flexible graphene pore wall. With more amounts of ions embedded in graphene pores, the pillar effect is more remarkable and a new one can be introduced more easily. In this way, upon repeated charging and discharging, pillar ions spread to all of the active surface area and the ion transport kinetics inside HOPC is greatly improved. This result was also confirmed by the real-time EIS study (Figure 3g); the Nyquist plots at low frequency region changed to be more vertical upon repeated cycling, reflecting the gradually enhanced ion transport inside HOPC. When the HOPC is fully activated, $\mathrm{Zn}^{2+}$ ion can easily enter the characteristic pores of HOPC upon partial dehydration (Figure $3 \mathrm{~h}, \mathrm{i}$ ). As a result, $\mathrm{Zn}^{2+}$ ion reaches all the active surface area inside HOPC, as revealed by the HAADFSTEM (Figure $3 \mathrm{j}$ ), and the EDS mappings of SEM and TEM (Supporting Information, Figures S26, S27). Notably, according to the molecular dynamics simulations, the prerequisite of this electrochemical activation process is that the chargecarrier ions can easily get into the characteristic pores upon partial dehydration, which in turn well illustrates the limited electrochemical activation effect of $\mathrm{Al}^{3+}$ ion based system.

Raman spectroscopy is an effective tool to reveal the properties of graphene-based materials and can reflect the characteristic features including doping, defects, stress, and the number of layers. ${ }^{[34]}$ For a graphene-based electrode material, its charging and discharging processes are intrinsically accompanied by the electrochemical doping of the graphene sheets in it. ${ }^{[35,36]}$ The positive doping and negative doping are divided by the zero charge potential (ZCP, ca. $0.4 \mathrm{~V}$ vs. $\mathrm{Ag} / \mathrm{AgCl}$ for $\mathrm{HOPC}$ in aqueous electrolytes). For example, when the applied potential is higher than ZCP, the graphene sheets in it are positively doped and vice versa. Both positive doping and negative doping will shift the Fermi level away from Dirac point and thus suppress the energy dissipation of phonons, finally inducing the intensity enhancements of $\mathrm{G}$ and D bands. ${ }^{[37-39]}$ Notably, the doping level of graphene sheets is closely related to the polarized degree of the electrode, and the rapid adsorption of counterions (relative to the polarity of electrode) can effectively compensate the polarization of the electrode and thus decreases the doping level of graphene sheets in it. In our case, owing to the compact microstructure of HOPC, counter ions may not reach the active sites as soon as possible when the electrode is polarized. Predictably, this effect shall be more remarkable when larger charge-carrier ions are utilized, hence enabling in situ Raman spectroscopy to be an effective technique to monitor the electrochemical behaviors of different ions inside HOPC electrode.

Figure $4 \mathrm{a}-\mathrm{c}$ show the contour-type in situ Raman spectra of fresh HOPC electrodes in different electrolyte; all the Raman spectra were collected by discharging the fresh electrode from open circuit potential (OCP, herein approximate to the $\mathrm{ZCP}$ ) to low potential, followed by a complete charging and discharging processes (Supporting Information, Figure S28). Taking the $\mathrm{Na}^{+}$ion based system as an example, when discharging fresh HOPC electrode from OCP (ZCP) to low potential, the Raman intensities gradually increase, corresponding to the negative doping process of the graphene sheets in HOPC (Figure 4a). The Raman intensities then gradually decrease when charging HOPC electrode from low potential to ZCP, indicating the de-doping process. Further charging HOPC electrode to high potential and then discharging back to ZCP do not bring about obvious changes on Raman intensities. This is mainly because the radius of counter ion $\left(0.335 \mathrm{~nm}\right.$ for $\mathrm{NO}_{3}{ }^{-}$ion $)$working in this potential range is smaller than the characteristic pore size of HOPC $(0.345 \mathrm{~nm})$, thus enabling counterions to compensate the 

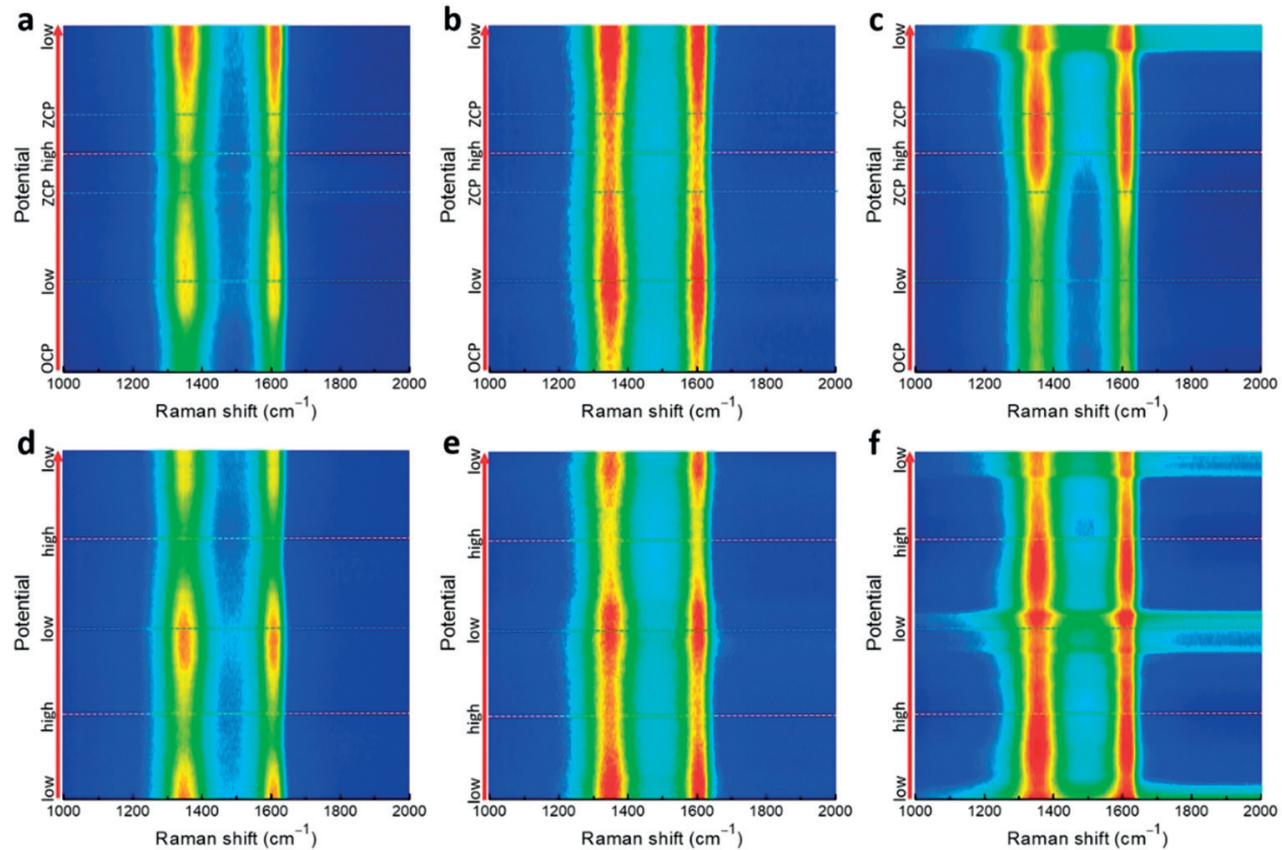

Figure 4. Contour-type in situ electrochemical Raman spectra of a)-c) fresh HOPC electrode and d)-f) activated HOPC electrode in different electrolytes: a),d) $\mathrm{NaNO}_{3}$; b),e) $\mathrm{Zn}\left(\mathrm{NO}_{3}\right)_{2}$; and c), f) $\mathrm{Al}\left(\mathrm{NO}_{3}\right)_{3}$, with red and blue contours showing the high and low Raman intensities, respectively.

polarization as fast as possible. Continuing to discharge HOPC electrode from ZCP to low potential again results in the intensity enhancements, showing the high reversibility of this electrochemical doping process. The $\mathrm{Zn}^{2+}$ ion based system shows a similar Raman response with that of $\mathrm{Na}^{+}$ion based system, except that the intensity enhancements are more remarkable when the HOPC electrode is polarized (Figure $4 \mathrm{~b}$ ), demonstrating the stronger interaction of $\mathrm{Zn}^{2+}$ ion with graphene pore walls during the electrochemical activation process. Interestingly, for $\mathrm{Al}^{3+}$ ion based system, there is no obvious Raman response variation when discharging fresh HOPC electrode from OCP (ZCP) to low potential and then charging back to ZCP (Figure $4 \mathrm{c}$ ), indicating that $\mathrm{Al}^{3+}$ ion cannot permeate fresh HOPC electrode. However, after experiencing the following positive polarization, that is, charging and discharging at the potential range between ZCP and high potential, obvious intensity enhancements are detected when discharging HOPC electrode to low potential. This result reflects that after activating by $\mathrm{NO}_{3}{ }^{-}$ion, $\mathrm{Al}^{3+}$ ion begins to slightly permeate HOPC upon negative polarizing. Notably, strong and broad background emerges at near low potential region, which is attributed to the luminescence from the recombination of thermalized electron-hole pairs induced by the excessive polarization. ${ }^{[40]}$ The above results further confirm the fact that trivalent $\mathrm{Al}^{3+}$ ion is too large to completely permeate the compact HOPC, and thus the divalent $\mathrm{Zn}^{2+}$ ion (or $\mathrm{Mg}^{2+}$ ion) is the most appropriate ionic charge carrier to achieve ultrahigh SCD in the HOPC electrode.

Contour-type in situ Raman spectra were also collected after the HOPC electrodes reached steady states, namely being fully activated upon repeated charging and discharging. As shown in Figure $4 \mathrm{~d}-\mathrm{f}$, the Raman responses of all three systems show high reversibility among the studied two consecutive cycles. When compared with the fresh state, $\mathrm{Na}^{+}$ion based system shows no obvious difference, but there is an intensity decline for $\mathrm{Zn}^{2+}$ ion based system. This phenomenon indicates that for $\mathrm{Na}^{+}$ion based system, it can reach steady state just at the first cycle owing to the relatively small size of $\mathrm{Na}^{+}$ion. But for $\mathrm{Zn}^{2+}$ ion based system, an activation process is needed to reach steady state, after which the interaction between $\mathrm{Zn}^{2+}$ ion and graphene pore wall will be appreciably declined, thus ensuring the fast transport of $\mathrm{Zn}^{2+}$ ion within HOPC electrode. However, for $\mathrm{Al}^{3+}$ ion based system, it seems that an effective activation cannot be achieved even upon repeated charging/discharging cycling, regarding the luminescence phenomenon still exists even after the system reaching steady state. ${ }^{[40]}$ These results are in agreement with above theoretical studies, and again support our conclusion that for compact HOPC, divalent $\mathrm{Zn}^{2+}$ ion (or $\mathrm{Mg}^{2+}$ ion) is the most superior ionic charge carrier to maximize the SCD in it.

Furthermore, we also took in situ Raman spectroscopy of $\mathrm{HOPC}$ electrode in $\mathrm{ZnSO}_{4}$ electrolyte; this electrolyte will be later used to construct full-cell zinc ion capacitor. Compared with the system using $\mathrm{Zn}\left(\mathrm{NO}_{3}\right)_{2}$ electrolyte, obvious intensity enhancements appear when HOPC electrode is positively polarized (Figure 5a,b), reflecting that $\mathrm{SO}_{4}{ }^{2-}$ ion has an unignorable interaction with graphene pore wall owing to its relatively large radius of $0.379 \mathrm{~nm}$. Also, after the HOPC electrode were fully activated and reached steady state in this electrolyte, the Raman intensities among both positive and negative polarizations show a decline (Figure $5 \mathrm{c}, \mathrm{d}$ ), consistent with previous results. Based on above results, we can generalize a conclusion that the ion with a size larger than the characteristic pores of the HOPC will induce obvious Raman 

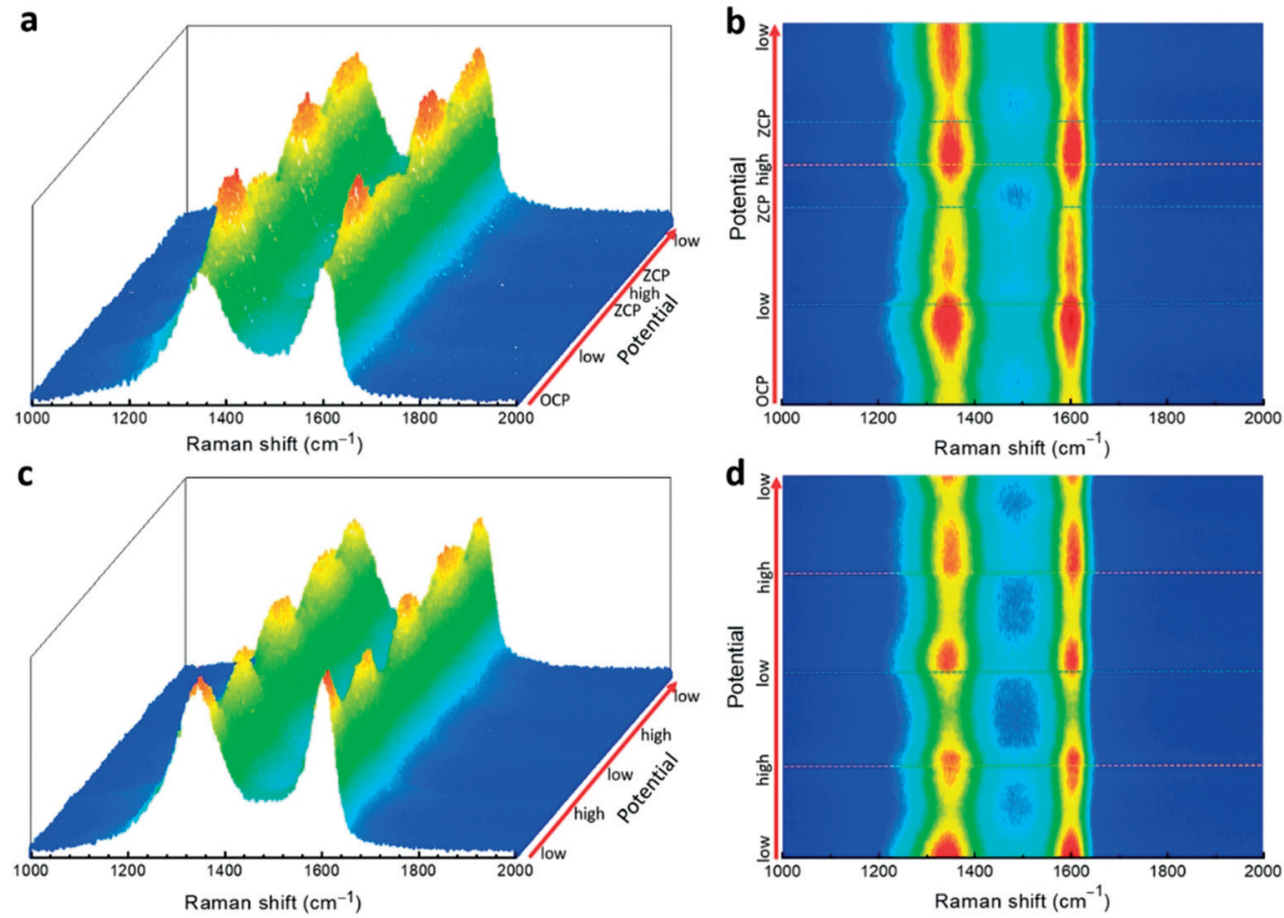

Figure 5. Contour-type in situ electrochemical Raman spectra of a),b) fresh $\mathrm{HOPC}$ electrode and c), d) activated $\mathrm{HOPC}$ electrode in $\mathrm{ZnSO}_{4}$ electrolyte, with red and blue contours showing the high and low Raman intensities, respectively.

intensity enhancements when they enter HOPC, regarding the size relationship of $\mathrm{NO}^{3-}$ ion $(0.335 \mathrm{~nm})<\mathrm{HOPC}$ $(0.345 \mathrm{~nm})<\mathrm{Na}^{+}$ion $(0.358 \mathrm{~nm})<\mathrm{SO}_{4}{ }^{2-}$ ion $(0.379 \mathrm{~nm})<$ $\mathrm{Zn}^{2+}$ ion $(0.430 \mathrm{~nm})<\mathrm{Al}^{3+}$ ion $(0.475 \mathrm{~nm})$ and their Raman responses.

Considering the high theoretical capacity $\left(820 \mathrm{mAhg}^{-1}\right)$, low redox potential $(-0.76 \mathrm{~V}$ vs. standard hydrogen electrode), high safety, and low cost of zinc-based electrochemistry, ${ }^{[41-43]}$ a zinc-ion hybrid capacitor (ZIC) was further constructed by using HOPC as the capacitive cathode and zinc foil as the battery-type anode (Supporting Information, Figure S29). Owing to the wide operating potential range of $\mathrm{HOPC}$ in aqueous electrolyte $(-0.8-0.8 \mathrm{~V}$ vs. $\mathrm{Ag} / \mathrm{AgCl})$, the HOPC based ZIC can work well within a wide voltage window of $0.2-1.8 \mathrm{~V}$ (Figure $6 \mathrm{a}$; Supporting Information, Figures S30-S32). The broad redox humps on the CV curves indicate the Faraday pseudo-capacitive reaction dominated charge storage mechanism of the HOPC based ZIC (Supporting Information, Figure S33). ${ }^{[21,44]}$ Even at a high scan rate of $20 \mathrm{mVs}^{-1}$, the $\mathrm{CV}$ curves show negligible shape distortion and the peak currents keep a linear relationship with scan rates, implying the excellent rate performance of HOPC based ZIC (Supporting Information, Figure S34). The galvanostatic charge/discharge (GCD) curves of HOPC based ZIC show symmetrical and quasi-triangular shapes, also demonstrating its pseudo-capacitive charge storage mechanism (Figure 6b).$^{[45]}$ Even at an ultrahigh current density of $20 \mathrm{Ag}^{-1}$, the GCD curve still maintains its symmetry with just a small $I R$ drop, again reflecting the excellent rate performance of HOPC based ZIC. The specific capacitance of HOPC based ZIC were further calculated from its GCD curves. At a current density of $0.1 \mathrm{Ag}^{-1}$, it delivers a high specific capacitance of $378 \mathrm{~F} \mathrm{~cm}^{-3}$ (or $168 \mathrm{mAh} \mathrm{cm}^{-3}$, Fig- ure $6 \mathrm{c}$ ), which is over four times higher than that of AC based ZIC $\left(88 \mathrm{~F} \mathrm{~cm}^{-3}\right.$ or $39 \mathrm{~mA} \mathrm{hm}^{-3}$; Supporting Information, Figure S35). When increasing the current density to $20 \mathrm{Ag}^{-1}$, HOPC based ZIC still delivers a high specific capacitance of $266 \mathrm{~F} \mathrm{~cm}^{-3}$ (or $118 \mathrm{~mA} \mathrm{~h} \mathrm{~cm}^{-3}$ ), with an outstanding capacitance retention over $70 \%$.

Benefiting from the ultrahigh volumetric capacitance and the wide operating voltage window, ZIC delivers excellent energy/power performances as revealed by the Ragone plot (Figure 6d). A superb energy density of $165 \mathrm{WhL}^{-1}$ $\left(112 \mathrm{Wh} \mathrm{kg}^{-1}\right)$ was achieved at a power density of $150 \mathrm{WL}^{-1}$ $\left(100 \mathrm{Wkg}^{-1}\right)$; even at an ultrahigh power density of $36 \mathrm{~kW} \mathrm{~L}^{-1}$ $\left(24 \mathrm{~kW} \mathrm{~kg}^{-1}\right)$, a high energy density of $120 \mathrm{WhL}^{-1}$ $\left(81 \mathrm{Wh} \mathrm{kg}^{-1}\right)$ was retained. These excellent energy/power performances make HOPC based ZIC be fully competitive to other types of state-of-the-art EES devices, such as electrochemical double layer capacitors (EDLCs), lead-acid batteries, nickel hydrogen (Ni-MH) batteries, nickel cadmium (Ni$\mathrm{Cd}$ ) batteries, hybrid capacitors, and even lithium-ion batteries (Figure 6d). ${ }^{[46]}$ Attractively, HOPC based ZIC also exhibits an excellent cycle stability with a capacity retention of about $100 \%$ upon 20000 charging/discharging cycles (Figure 6e). Moreover, considering the electrodes with large areas and high areal mass loadings are highly desired for practical applications, ${ }^{[43,47,48]}$ a pouch-cell device was assembled (Figure $6 \mathrm{f}, \mathrm{g}$ ). Even with a large area of $4 \times 6 \mathrm{~cm}^{2}$ and a high areal mass loading of $10 \mathrm{mg} \mathrm{cm}^{-2}$, the pouch-cell ZIC delivers a high volumetric capacity of $149 \mathrm{mAh} \mathrm{cm}^{-3}$ at $1 \mathrm{Ag}^{-1}$, and can work well throughout 1000 charging/discharging cycles (Figure 6h). This result again highlights the attractiveness of HOPC based ZIC for practical applications. 

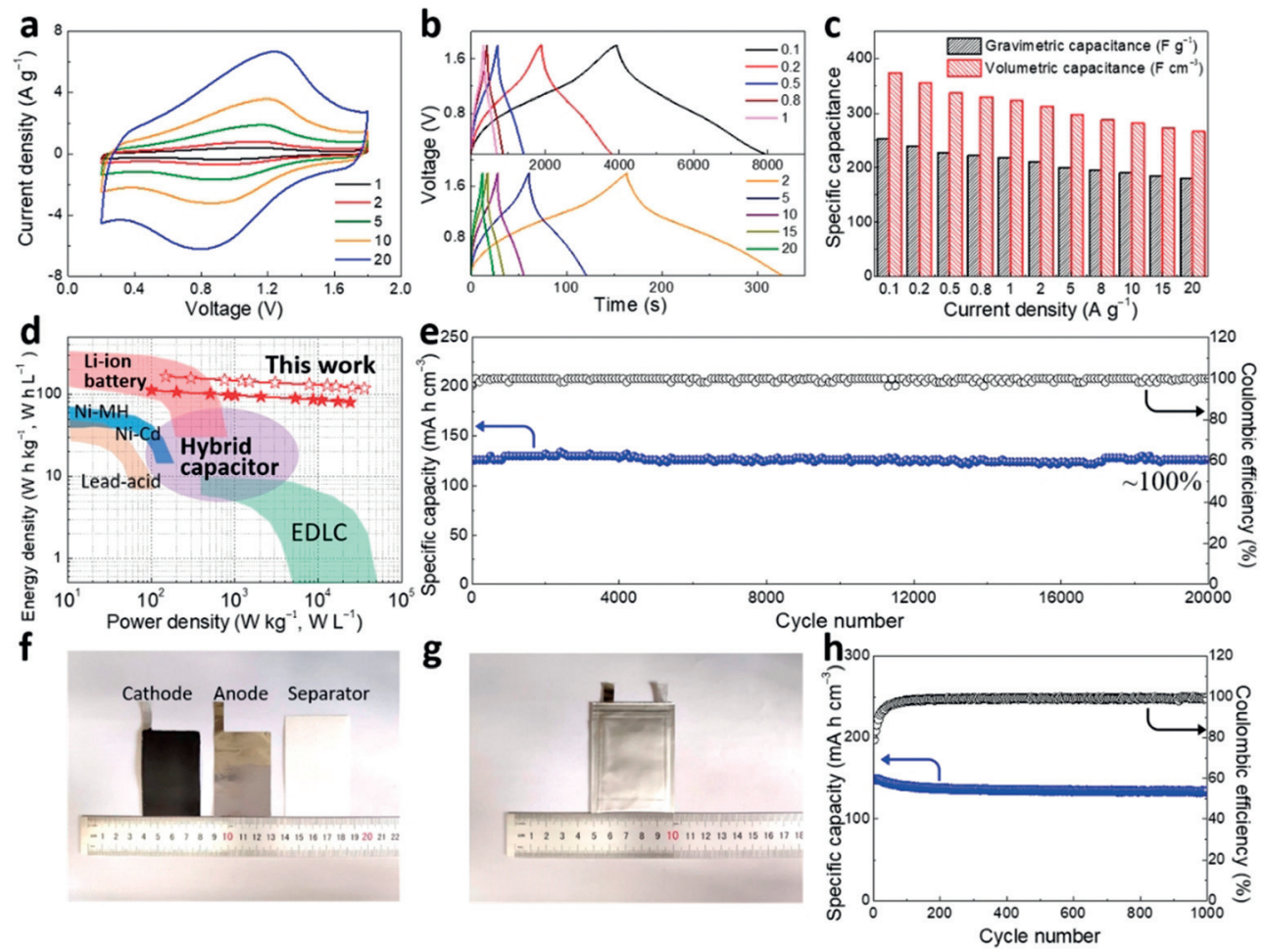

Figure 6. Electrochemical performances of HOPC based ZIC. a) CV curves at different scan rates $\left(\mathrm{mV} \mathrm{s}^{-1}\right)$. b) GCD curves at different current densities $\left(\mathrm{Ag}^{-1}\right)$. c) Gravimetric and volumetric capacitances at different current densities. d) Comparation of energy/power densities of HOPC based ZIC with those of other state-of-the-art EES devices. e) Long-term cycling stability of HOPC based ZIC at the current density of $5 \mathrm{Ag}^{-1}$. f) Digital photograph of the HOPC cathode, zinc foil anode, and filter paper separator for the pouch-cell ZIC. g) Digital photograph of the assembled pouch-cell ZIC. h) Long-term cycling stability of the pouch-cell ZIC at the current density of $1 \mathrm{Ag}^{-1}$.

\section{Conclusion}

A SCD maximization approach is demonstrated to achieve highly efficient capacitive energy storage with both high energy density and high power density. In this case, partially dehydrated divalent $\mathrm{Zn}^{2+}$ ions were densely and neatly packed in a highly ordered and compact scaffold, namely HOPC, achieving an unparalleled spatial charge density of about $550 \mathrm{Ccm}^{-3}$. The electrochemical behaviors of different charge-carrier ions in HOPC and the superiority of divalent $\mathrm{Zn}^{2+}$ ion were comprehensively revealed by Monte Carlo calculations, molecular dynamics simulation, and in situ electrochemical Raman spectroscopy. On these bases, a fullcell zinc ion capacitor was constructed that delivers simultaneous high-energy and high-power performances, making it be fully competitive to other state-of-the-art EES devices and thus opening up the door of high-energy-density capacitive energy storages. Also, for the first time, in situ Raman spectroscopy was employed to reveal the interaction between charge-carrier ions and electrode materials, greatly enriching the community of in situ characterization techniques for supercapacitors.

\section{Acknowledgements}

We acknowledge the financial support from the National Key R\&D Program of China (2016YFA0200200,
2017YFB1104300), NSFC (No. 51673026, 51433005, 21674056), NSFC-MAECI (51861135202).

\section{Conflict of interest}

The authors declare no conflict of interest.

Keywords: capacitive energy storage .

in situ Raman spectroscopy · ion sieving effect .

spatial charge density $\cdot$ zinc ion hybrid capacitor

[1] H. Sun, L. Mei, J. Liang, Z. Zhao, C. Lee, H. Fei, M. Ding, J. Lau, M. Li, C. Wang, X. Xu, G. Hao, B. Papandrea, I. Shakir, B. Dunn, Y. Huang, X. Duan, Science 2017, 356, 599-604.

[2] M. Zhang, X. Yu, H. Ma, W. Du, L. Qu, C. Li, G. Shi, Energy Environ. Sci. 2018, 11, 559-565.

[3] C. Yang, J. Chen, X. Ji, T. P. Pollard, X. Lu, C.-J. Sun, S. Hou, Q. Liu, C. Liu, T. Qing, Y. Wang, O. Borodin, Y. Ren, K. Xu, C. Wang, Nature 2019, 569, 245-250.

[4] P. Simon, Y. Gogotsi, B. Dunn, Science 2014, 343, 1210-1211.

[5] K. Xu, Chem. Rev. 2004, 104, 4303-4417.

[6] K. Xu, Chem. Rev. 2014, 114, 11503-11618.

[7] C. Merlet, B. Rotenberg, P. A. Madden, P.-L. Taberna, P. Simon, Y. Gogotsi, M. Salanne, Nat. Mater. 2012, 11, 306-310.

[8] M. Salanne, B. Rotenberg, K. Naoi, K. Kaneko, P. L. Taberna, C. P. Grey, B. Dunn, P. Simon, Nat. Energy 2016, 1, 16070.

[9] Y. Gogotsi, P. Simon, Science 2011, 334, 917-918. 
[10] Y. Zhu, S. Murali, M. D. Stoller, K. J. Ganesh, W. Cai, P. J. Ferreira, A. Pirkle, R. M. Wallace, K. A. Cychosz, M. Thommes, D. Su, E. A. Stach, R. S. Ruoff, Science 2011, 332, 1537-1541.

[11] M. R. Lukatskaya, S. Kota, Z. Lin, M.-Q. Zhao, N. Shpigel, M. D. Levi, J. Halim, P.-L. Taberna, M. Barsoum, P. Simon, Y. Gogotsi, Nat. Energy 2017, 2, 17105.

[12] L. Zhang, X. Yang, F. Zhang, G. Long, T. Zhang, K. Leng, Y. Zhang, Y. Huang, Y. Ma, M. Zhang, Y. Chen, J. Am. Chem. Soc. 2013, 135, 5921 - 5929 .

[13] W. Lee, S. Muhammad, C. Sergey, H. Lee, J. Yoon, Y.-M. Kang, W.-S. Yoon, Angew. Chem. Int. Ed. 2020, 59, 2578-2605; Angew. Chem. 2020, 132, 2598-2626.

[14] K. Sheng, Y. Sun, C. Li, W. Yuan, G. Shi, Sci. Rep. 2012, 2, 247.

[15] X. Wang, L. Lv, Z. Cheng, J. Gao, L. Dong, C. Hu, L. Qu, Adv. Energy Mater. 2016, 6, 1502100.

[16] B. Lu, F. Liu, G. Sun, J. Gao, T. Xu, Y. Xiao, C. Shao, X. Jin, H. Yang, Y. Zhao, Z. Zhang, L. Jiang, L. Qu, Adv. Mater. 2020, 32 1907005.

[17] Y. Wang, Y. Song, Y. Xia, Chem. Soc. Rev. 2016, 45, 5925-5950.

[18] A. C. Forse, C. Merlet, J. M. Griffin, C. P. Grey, J. Am. Chem. Soc. 2016, 138, $5731-5744$.

[19] M. Wu, F. Chi, H. Geng, H. Ma, M. Zhang, T. Gao, C. Li, L. Qu, Nat. Commun. 2019, 10, 2855.

[20] B. Yao, J. Chen, L. Huang, Q. Zhou, G. Shi, Adv. Mater. 2016, 28, $1623-1629$.

[21] H. Ma, H. Geng, B. Yao, M. Wu, C. Li, M. Zhang, F. Chi, L. Qu, ACS Nano 2019, 13, 9161 - 9170.

[22] M. Song, H. Tan, D. Chao, H. J. Fan, Adv. Funct. Mater. 2018, 28 , 1802564

[23] M.-C. Lin, M. Gong, B. Lu, Y. Wu, D.-Y. Wang, M. Guan, M. Angell, C. Chen, J. Yang, B.-J. Hwang, H. Dai, Nature 2015, 520, $324-328$.

[24] M. Thommes, K. Kaneko, A. V. Neimark, J. P. Olivier, F. Rodriguez-Reinoso, J. Rouquerol, K. S. W. Sing, Pure Appl. Chem. 2015, 87, 1051-1069.

[25] J. Zhao, H. Lai, Z. Lyu, Y. Jiang, K. Xie, X. Wang, Q. Wu, L. Yang, Z. Jin, Y. Ma, J. Liu, Z. Hu, Adv. Mater. 2015, 27, $3541-$ 3545 .

[26] H. Ma, C. Li, M. Zhang, J.-D. Hong, G. Shi, J. Mater. Chem. A 2017, 5, 17040-17047.

[27] J. Chmiola, G. Yushin, Y. Gogotsi, C. Portet, P. Simon, P. L. Taberna, Science 2006, 313, 1760-1763.

[28] J. Chmiola, C. Largeot, P.-L. Taberna, P. Simon, Y. Gogotsi, Angew. Chem. Int. Ed. 2008, 47, 3392-3395; Angew. Chem. 2008, 120, 3440 - 3443 .

[29] M. Deschamps, E. Gilbert, P. Azais, E. Raymundo-Pinero, M. R. Ammar, P. Simon, D. Massiot, F. Beguin, Nat. Mater. 2013, 12 , $351-358$.
[30] C. Pean, B. Daffos, B. Rotenberg, P. Levitz, M. Haefele, P.-L. Taberna, P. Simon, M. Salanne, J. Am. Chem. Soc. 2015, 137, $12627-12632$

[31] C. Prehal, C. Koczwara, N. Jaeckel, A. Schreiber, M. Burian, H. Amenitsch, M. A. Hartmann, V. Presser, O. Paris, Nat. Energy 2017, 2, 16215 .

[32] M. Carrillo-Tripp, H. Saint-Martin, I. Ortega-Blake, Phys. Rev. Lett. 2004, 93, 168104.

[33] R. Futamura, T. Iiyama, Y. Takasaki, Y. Gogotsi, M. J. Biggs, M. Salanne, J. Segalini, P. Simon, K. Kaneko, Nat. Mater. 2017, 16, $1225-1232$

[34] A. C. Ferrari, D. M. Basko, Nat. Nanotechnol. 2013, 8, 235-246.

[35] A. Das, S. Pisana, B. Chakraborty, S. Piscanec, S. K. Saha, U. V. Waghmare, K. S. Novoselov, H. R. Krishnamurthy, A. K. Geim, A. C. Ferrari, A. K. Sood, Nat. Nanotechnol. 2008, 3, 210-215.

[36] M. Bruna, A. K. Ott, M. Ijaes, D. Yoon, U. Sassi, A. C. Ferrari, ACS Nano 2014, 8, 7432-7441.

[37] J. Yan, Y. Zhang, P. Kim, A. Pinczuk, Phys. Rev. Lett. 2007, 98 , 166802.

[38] M. Kalbac, A. Reina-Cecco, H. Farhat, J. Kong, L. Kavan, M. S. Dresselhaus, ACS Nano 2010, 4, 6055-6063.

[39] M. Kalbac, H. Farhat, J. Kong, P. Janda, L. Kavan, M. S. Dresselhaus, Nano Lett. 2011, 11, 1957-1963.

[40] F. Herziger, R. Mirzayev, E. Poliani, J. Maultzsch, Phys. Status Solidi B 2015, 252, 2451-2455.

[41] F. Wang, O. Borodin, T. Gao, X. Fan, W. Sun, F. Han, A. Faraone, J. A. Dura, K. Xu, C. Wang, Nat. Mater. 2018, 17, 543-549.

[42] J. Zheng, Q. Zhao, T. Tang, J. Yin, C. D. Quilty, G. D. Renderos, X. Liu, Y. Deng, L. Wang, D. C. Bock, C. Jaye, D. Zhang, E. S. Takeuchi, K. J. Takeuchi, A. C. Marschilok, L. A. Archer, Science 2019, 366, 645-648.

[43] G. Sun, H. Yang, G. Zhang, J. Gao, X. Jin, Y. Zhao, L. Jiang, L. Qu, Energy Environ. Sci. 2018, 11, 3367-3374.

[44] H. Ma, Q. Zhou, M. Wu, M. Zhang, B. Yao, T. Gao, H. Wang, C. Li, D. Sui, Y. Chen, G. Shi, J. Mater. Chem. A 2018, 6, 6587-6594.

[45] H. Ma, D. Kong, Y. Xu, X. Xie, Y. Tao, Z. Xiao, W. Lv, H. D. Jang, J. Huang, Q.-H. Yang, Small 2017, 13, 1701026.

[46] W. Zuo, R. Li, C. Zhou, Y. Li, J. Xia, J. Liu, Adv. Sci. 2017, 4, 1600539.

[47] X. Wang, Y. Ding, F. Chen, H. Lu, N. Zhang, M. Ma, ACS Appl. Energy Mater. 2018, 1, 5024-5032.

[48] X. Wang, Y. Ding, H. Lu, F. Chen, N. Zhang, M. Ma, Chem. Eng. J. 2018, 347, 754-762.

Manuscript received: April 11, 2020

Revised manuscript received: June 4, 2020

Accepted manuscript online: June 7, 2020

Version of record online: July 7, 2020 